https://doi.org/10.48009/2_iis_2008_330-336

\title{
ENTERPISE CONTENT MANAGEMENT: A USABILITY STUDY
}

\author{
Robert L. Totterdale, Robert Morris University, robert.totterdale@accenture.com
}

\begin{abstract}
Over $90 \%$ of business records today are available in electronic form. With vast increases in electronic business content being generated and received daily, companies must now consider new approaches for organizing and categorizing their content (e.g. emails, documents, pdfs) to meet operational and compliance needs. Enterprise Content Management (ECM) Software has been suggested as a solution to address these needs. This paper develops a framework for the usability testing of a commercial ECM product being evaluated by a large professional services firm. Using this framework, the study assesses five key dimensions of usability including effectiveness, efficiency, satisfaction, ease of use and performance. The study is qualitative.
\end{abstract}

Keywords: E-records, Email, ECM, Information Technology (IT), Personal Information Management, Collaboration, E-discovery

\section{INTRODUCTION}

With over 90\% of business records now being available in electronic form, organizations must begin to implement new technologies, policies and procedures to effectively capture, organize and maintain their content. The legal importance of accurately defined and maintained electronic content can be seen in the Federal Rules of Civil Procedure enacted in December 2006 which requires that organizations preserve and produce Electronic Stored Information at the request of the courts [15]. The failure by an organization to respond to court requests (i.e. e-discovery) for information can result in substantial penalties and fines as evidenced by recent judgments of $\$ 10$ million against the Bank of America and \$29.3 million against UBS Warburg [16].

In addition to meeting legal and regulatory requirements, organizations are also beginning to understand the importance of maintaining their electronic content to support communications, knowledge management and collaboration needs for individuals located around the globe. Inconsistently or improperly filed electronic content impedes an individual's ability to collaborate and to conduct business in an efficient manner. Poorly managed content also leads to the storage of duplicate content and can result in higher operating costs relating to increased storage requirements and disaster recovery needs.

Solutions to the problem of content management are now being offered by independent software vendors as well as the major infrastructure vendors such as IBM, Microsoft, EMC and Oracle. These solutions, often referred to as Enterprise Content Management (ECM) products, provide comprehensive support for profiling (organizing), retrieving and searching, and managing large data stores that house millions of electronic documents and emails.

This paper summarizes the results of a usability test for an ECM product conducted at a large professional services organization. Several key measures of usability including effectiveness, efficiency, satisfaction, ease of use and performance were assessed.

Four primary research questions (problems) addressed in this paper include: "Does the current release of the product contain usability flaws that prevent completion of common ECM tasks? What obstacles prevent the completion of key content management functions? What concerns do users have regarding the use of the technology? Do users consider the product easy to use for profiling of documents and emails?

\section{Literature Review}

A review of the literature begins with a definition of usability testing by Rubin [13] who suggests that it is a "process that employs participants who are representative of the target population to evaluate the degree to which a product meets specific usability criteria” (p. 25). Using key measures from the International Organization for Standards (ISO), Jones and Teevan [9] argue that usability assessment criteria should include effectiveness, efficiency, satisfaction, ease of use and performance. Each of these was considered in the testing process.

Since the usability testing process addresses how content is categorized, organized and subsequently 
retrieved, the fields of human computer interaction (HCI), personal information management [9], knowledge management [3], group information management [8], library sciences, and records management were reviewed. A summary of the key finding are included in the following paragraphs.

Beginning with content categorization, a common technique found in information sciences is to assign metadata to each item to be saved. Metadata is defined by the National Information Standard Organization as "structured information that describes, explains, locates or otherwise makes it easier to retrieve, use or manage an information resource" [4]. Organizations have used both manual and automated approaches to assign metadata to content with various adoption and success rates. No one approach has proven to be $100 \%$ accurate and able to fully meet the operational and compliance needs of an organization. Manual classification approaches such as key entry, the use of folksonomies [10], corporate taxonomies and controlled lists [11] have also been used to tag items for subsequent retrieval.

Alternative automated approaches for categorizing content such as using defaults, dragging and dropping of content into folders and auto-creation of metadata have been attempted for certain content types with varying degrees of success [9]. Machine learning and agent based techniques for automatically organizing emails has not found broad acceptance by users since the recommended classifications may not always agree with the users intentions. Other issues exist with automated filing of content into new categories (versus old ones) since the system has no way of knowing where to place the content. Palen [12] cites another concern relating to the use of defaults for categorization indicating that $81 \%$ of users never change their default settings even though there may have been changes to some of the values.

Prior to the act of categorizing content, however, individuals must first make a decision to keep the content. From the field of knowledge management, Davenport and Prusack [3] have argued that trust, reward systems, value systems and vocabularies along with other cultural differences influence a person's decision making actions. Others such as Goffman [6] and Hofstede and Hofstede [7] have reached similar conclusions. Privacy and security are two other factors that have been identified that influence a user's decision making process relating to content categorization.
Once a decision is made to store content electronically, a method for organizing the content must be determined. Research has found that users have different styles and approaches for creating folder structures with some creating a few while others create many [9]. In addition, it has been found that the more folders a user has the more likely they are to generate failed folders (i.e. those with only one or two elements). In a comprehensive review of personal information management, Jones [8] cites a number of findings including that the filing of content is a cognitively difficult task, that the act of filing requires the user to be able to anticipate future needs, and that the forms and volume of content also influence an individual's ability to organize information. The fact that categories change over time also complicates the filing process particularly when automated techniques are attempted.

Special issues exist relating to the organizing of emails since users 'feel overwhelmed by the volume of messages and concerned about processing incoming messages effectively" [1]. Jones and Teevan [9] highlight key issues with email including difficulties with organizing the messages, the amount of attention required given the volumes involved and the complexity of the filing decision making process. Other complexities arise when groups of individuals must share the emails.

Having addressed both the decision to file content and approaches for organizing content, the ability to retrieve content must also be assessed. With enhanced search capabilities, some suggest that organizing information is no longer necessary [2] while others argue that it is essential. Browsing versus searching remains many users preferred way to access personal information, even when search is available. Other research cited by Jones and Teevan [9] suggests that "individual finding strategies do not work for a group" (p. 243) and that differences in individual styles can influence behaviors.

Lastly, a case study from an organization that has implemented an ECM product has reported that the initial adoption rates for email filing ranged from $1 \%$ to $74 \%$ depending on the operating location [5]. This finding raises questions about the many technical and non-technical factors that can influence the proper maintenance of electronic records in an organization.

\section{Organizational Context}

The organization in the study is a professional services firm with over 1000 employees. The firm conducts a large number of projects in a number of 
industries on an annual basis. As a result of their business activity, the firm must cope with growing document and email repositories that currently contain millions of records. Individual practitioners receive and send between 50 and 400 emails daily and routinely create, modify and access a large number of electronic records. Many individuals have set up personal (non-shared) file folders in Outlook for email storage and retrieval and use a commercial document management system for filing and sharing documents. When creating new content, most individuals also input a profile (e.g. metadata) for each document that includes information such as client, project and sub-folder.

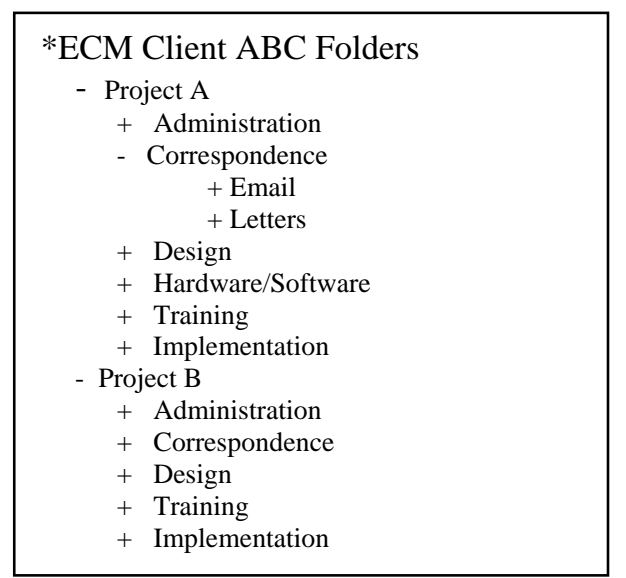

Because of the need to more effectively share content (e.g. email and documents) within the organization and to be able to meet compliance and regulatory needs, the firm decided to evaluate a leading commercial ECM product by performing a usability test on selected product capabilities. The solution combined both email and documents into a single repository, provided for enhanced profiling capabilities using a "folder metaphor" in Outlook and supported the retrieval of content by folder or through a full text search. A representation of a folder structure and its related metadata is provided below in Figure 1.

Figure 1. Folder Structure and Metadata

\section{RESEARCH METHODOLOGY}

A usability testing methodology identified by Rubin [13] provided the framework for conducting this study. Considered to be a user centered methodology, this approach recognizes the need for user input throughout the process. Other studies [17] have successfully used this approach to validate the usability of web sites. The steps in the methodology included defining test objectives, developing the test plan, selecting and acquiring participants, preparing test materials, conducting the test, debriefing the participants and developing recommendations.

The primary objective for the test was to assess the usability of the ECM product for the organization. A test plan was developed that identified the need to set up a lab environment and a six week testing timeframe.

Management selected one of their larger practice groups that were heavy users of email and electronic records to represent the organization in the testing process. A total of 12 users were selected by the leader of the practice group to participate. This number was chosen based on Spyridaki's [14] argument that a true experimental design requires a minimum of 10-12 users per condition. The users represented a cross section from the practice that included eight executives and four support personnel. All of the participants involved had experience with the filing of email in folders in Outlook as well as with the profiling/filing of documents in the existing document management system.

After participants were selected, testing and training materials were developed and a test environment was established. Survey documents were developed to obtain user responses regarding their experience in processing transactions in the ECM software. In addition, a test environment was established that utilized a converted copy of a large database of documents currently in use by the participants along with a copy of their individual Outlook mailboxes. 
By providing electronic content that was familiar to the participants and of production size database volumes, it was believed that a more realistic assessment of the products capabilities could be obtained. A full text search index and a series of client/project folders were also created for use in the test.

Upon completion of the test preparation process, an orientation session was held with all participants to review the objectives of the testing process and to provide them an overview of the system and its key processes. This was the first step in the conduct of the test. After the orientation session, each participant was asked to visit the test lab where they were individually given a brief training guide on the new product along with instructions for completing four transactions. The four transactions included profiling an email, profiling a document, profiling an email with attachments and performing searches. A monitor was assigned to the lab to observe the participants, to provide assistance and to answer any questions. Users were then asked to return to their offices, to process transactions on their own and to respond to an electronic survey based on their experiences.

During the testing process, observations were recorded by the monitor regarding how individuals interacted with the system. Once the lab sessions were completed, all of the participants were asked to attend one of two scheduled focus group sessions where additional questions were asked to gain a better understanding of how the ECM product would support the organizations needs. At the conclusion of the focus group sessions, a summary of recommendations and issues relating to the ECM product were prepared for review with management.

\section{RESULTS}

All twelve participants responded to an electronic survey and attended one of two focus group sessions that were held. Feedback was obtained regarding their experience in profiling/filing of electronic documents and email, in searching and retrieving content and in securing the content that was entered. To satisfy the primary objective of the usability test, the participants were also asked their preference about acquiring the new ECM solution versus staying with their existing document management technology (e.g. a comparative assessment). The results which follow have been summarized into five major categories of usability including effectiveness, efficiency, ease of use, satisfaction, and performance.
The effectiveness of the product was measured by counting the number of participants who were able to successfully complete all four of the scripted transactions. Based on observations and feedback, 10 of the 12 participants were able to complete the transactions for an overall score of $83 \%$. Two of the participants had difficulty with the user interface and were unable to initially file email transactions or retrieve a specific document.

The next category of usability was efficiency. The primary measure of efficiency used for this study was the relative amount of time that it took to file or profile an item in the test system versus saving it in Outlook or the document management system. All respondents felt that it was somewhat less efficient than using the existing systems. This inefficiency was due to the system requiring additional "clicks" and steps to accomplishing a task as well as the fact the filing act took slightly longer in the test system than what they were accustomed to in Outlook.

Ease of use was measured based on user responses from the survey reflecting their test experience. From the survey, $75 \%$ agreed that it was easy to file and retrieve documents in the system, $75 \%$ felt that profiling would make it easier to share documents with others, $66 \%$ felt that the software provided a good mechanism to secure content and $66 \%$ felt that it was easy to file and retrieve emails. With respect to search, $100 \%$ of the responses felt that it was easy to locate documents and emails using the new full text search capabilities. One of the participant subgroups, the professionals, found the system to be easier to use than the support personnel.

Another measure obtained from the survey was the user's overall satisfaction with the product and an indication whether they believe it should be adopted in the organization. In this regard 9 out of 12 (75\%) felt it was an improvement over the existing system while 10 out of 12 (83\%) agreed that the organization should proceed with implementing the new ECM solution.

The final measure of usability related to performance. From direct observation of user activities and subsequent written feedback, the following performance characteristics were noted. Routine transactions while somewhat slower than the current system processed in an acceptable timeframe. Some initial slowdowns occurred during the start-up of the application for a user, but improved once the user was in the application. Three issues relating to system response were also noted including; search results occasionally taking more than 3 minutes, 
delays between filing content and when the content appears in the full text search, and the length of time it took to file groups of emails ( 20-30 seconds) versus a single email.

In addition to the specific measures of usability summarized above, the participants also identified a number of key benefits and potential obstacles regarding the profiling of emails and documents and the product's search capabilities. With respect to benefits, it was believed that the product would provide for improved sharing and collaboration with others and through the search mechanism would allow users to find content much more easily. Another benefit cited was the ability to have both emails and documents stored and accessible from a single repository thus improving access to content. In contrast to the value and benefits from the product, a number of obstacles or barriers to its usage were also noted as indicated in the following paragraph.

One major obstacle involved the cluttered appearance of the Outlook Interface when a user had a large number of projects or folders that were in use. Participants expressed concerns about whether users would bother to file content when faced with complex or lengthy tree structures. They also felt that it was likely that errors would be made in filing content if there were many folders or subfolders in use. Another obstacle related to user concerns about the incremental effort required to set up folders in the product versus in Outlook. Although the product provided new capabilities that were valued, the incremental effort was viewed as a barrier to usage. Differences in the search approach for the ECM product and Outlook were also identified as a potential source of confusion. In addition to product differences, there were differences noted amongst users regarding their perception of the need to profile content, the way in which they label/describe content, and their views on the sharing of content. Each of these could impact how and if a user chooses to work with the product.

Also, although the product has been in the market for some time, software bugs were identified by the users while testing the product that although minor in nature impacted the quality of the user experience. Lastly, users expressed concerns about the confidentiality and accessibility of content. Although they understood how to secure documents, concerns remained about others being able to view or edit highly confidential materials.

\section{CONCLUSIONS}

ECM vendors are combining powerful search technologies with integrated email and electronic record repositories to facilitate the filing and categorization of content for subsequent access and retrieval. Allowing users the ability to categorize their content through a familiar Outlook interface and folder structure has provided both opportunities and challenges as the software vendors attempt to respond to the huge increases in electronic content being processed on a daily basis.

In this research, a usability test was conducted on a popular ECM product to determine its suitability for use in a professional services organization, The results of the test indicated an overall $80 \%$ satisfaction with the product and concurrence that the product would be an improvement over the use of the current document management system by offering new search and email profiling capabilities. However, challenges remained with its adoption and usability.

A number of the obstacles that were observed or reported through survey responses were consistent with findings from the literature. One obstacle was the overwhelming volume of electronic content that competes for a user's attention and increases the complexity and frequency of filing. Further, when filing content users were faced with decisions on where to file (e.g. complex folder structures), when to file, and how to file (manually, automated and with whom I should file it) each of which can introduce errors into the filing and categorization process. In addition, even though the ECM product provided security capabilities, users remained concerned about filing highly confidential information into a shared repository.

Another obstacle observed was that users have a mental model of how applications should operate, particularly ones that use Outlook as a primary interface. Because the ECM product performed certain functions differently than what they were accustomed to in Outlook, some users became confused as they attempted to complete transactions.

In addition to preferring to see user interfaces consistent with Outlook, users also require reasonable system performance when using a product. Although the users were able to work through the issues relating to system response time in the test, it is questionable if they would accept long response times on content searches in a production environment. Since the users had limited search 
capabilities in their current environment, even a slow search in this instance was deemed to be beneficial.

Based on the research, the users who are most likely to use the ECM product are those with a small number of projects (e.g. places to file content), have a need or interest in sharing content with others, and who either know how to secure content or are not concerned with the sharing of content of others. Another characteristic of users who would likely use the system are those users that find value in saving content either for themselves or others for subsequent access and retrieval.

Conversely, for individuals or groups that have large numbers of concurrent projects that they are working on, profiling of content through an outlook "folder" structure may be difficult and may result in the incorrect filing of content in the wrong folder. Their adoption of the product, particularly in the area of email filing would be predicted to be lower or of lower accuracy than the other user group previously identified.

The implications of the research are three fold. First, for users with limited numbers of folders and or low volumes of content to be filed, the product represents a good productivity tool for keeping, maintaining, securing and retrieving electronic content. Second, users that have a large number of places to file content (e.g. folders) can take advantage of the product but may need to have some incentives and or automated tools in place for them to fully adopt the solution. Thirdly, ECM product vendors can use these finding to help improve the usability of their solutions. Specific areas of focus include improvements to system performance, implementing more consistency within Outlook and their application, developing more efficient mechanisms for the filing/profiling of content, and improving means to easily and more fully secure the electronic content.

With 12 participants and a limited scope, opportunities exist for further research. Other dimensions such as globalization and culture, legal and regulatory implications, policy, and data fragmentation could be examined to assess their impact both on a product and on an individual's likelihood of properly filing electronic content. In addition, the influence of the organization's standards and practices could be evaluated to understand how it impacts the ECM product adoption. In addition to the measurement of usability in this study, research could also be performed to examine actual adoption rates for the filing of different types of content to determine other approaches for improving compliance and adoption. Further examination of differences in product adoption due to role in the organization, age, and practice area may also provide insights regarding how to improve usability.

In summary, although a majority of the usability measures assessed in this study suggested a 66-80\% level of satisfaction by the users, work remains to improve the usability of the product to address some of the major obstacles that can influence adoption. In today's increasingly electronic world, it will be critical for organizations to both understand and resolve the many technical and non-technical factors that impede the proper filing and management of electronic content.

\section{REFERENCES}

1. Bellotti, V., Ducheneaut, N., Howard, M., Smith, I., \& Grinter, R. (2005). Quality vs. Quantity: Email centric task-management and its relation with overload. Human-Computer Interaction, 20, 89-138.

2. Cutrell, E., Dumais, S., \& Teevan, J. (2006). Searching to eliminate personal information management. Communications of the ACM, 49(1), 58-64.

3. Davenport, T., \& Prusak, L. (1998). Working Knowledge. Boston: Harvard Business School Press.

4. Franks, P., \& Kunde, N. (2006). Why METADATA Matters. Information Management Journal, 40(5), 55.

5. Freese, A. (2007). How Livelink ECM-eDOCS DM Does Email. Paper presented at the Summit 2007.

6. Goffman, E. (1959). The presentation of self in everyday life. New York: Anchor-Doubleday.

7. Hofstede, G., \& Hofstede, G. J. (2005). Cultures and Organizations : Software of the Mind. New York: McGraw-Hill.

8. Jones, W. (2008). Keeping Found Things Found. Burlington: Morgan Kaufmann Publishers.

9. Jones, W., \& Teevan, J. (2007). Personal Information Management. Seattle: University of Washington Press. 
10. Lubbes, R. K. (2003). So you want to implement automatic categorization? Information Management Journal, 37(2), 60.

11. O'Kane, T. (2006). United by a Common Language: Developing a Corporate Taxonomy. Information Management Journal, 40(4), 58.

12. Palen, L. (1999). Social, individual and technological issues for groupware calendar systems. Paper presented at the SIGCHI Conference on Human Factors in Computing Systems: The CHI is the Limit, New York.

13. Rubin, J. (1994). Handbook of Usability Testing. New York: John Wiley \& Sons, Inc.
14. Spyridakis, J. H. (1992). Conducting Research in Technical Communications: The Application of True Experimental Designs. Technical Communications, 607-624.

15. Swart, R. S., \& Erbacher, R. F. (2007). Legal Developments in eDiscovery: Implications for Security Management. Utah State University.

16. Volonino, L., Sipior, J. C., \& Ward, B. T. (2007). Managing the Lifecycle of Electronically Stored Information. Information Systems Management, 24(3), 231-238.

17. Ward, J. L. (2006). Web site redesign: the University of Washington Libraries' experience. OCLC Systems and Services, 22(3), 207-216. 